\title{
Étude comparative des produits d'hydrolyse enzymatique, et, catalytique du lactosérum déprotéiné sur résines échangeuses d'ions
}

\author{
par \\ M. DEMAIMAY \\ Laboratoire de Chimie-Biochimie Alimentaire \\ Ecole Nationale d'Ingénieurs des Techniques des Industries \\ Agro-Alimentaires \\ La Géraudière - 44072 Nantes Cedex
}

\section{INTRODUCTION}

L'accroissement important des quantités de lactosérum de fromagerie produit ces dernières années en France et en Europe conduit de plus en plus vers un manque de débouché pour ce produit. En effet, les faibles propriétés fonctionnelles, organoleptiques et techniques du lactose à savoir : le phénomène d'intolérance [1-2], son faible pouvoir sucrant et sa cristallisation rapide, sont des handicaps certains pour la valorisation directe du lactosérum en alimentation humaine. Au contraire, les procédés d'hydrolyse de ce dernier conduisent à des produits qui grâce à la présence des monosaccharides : glucose et galactose, possèdent de bonnes propriétés.

Ainsi, la transformation du lactose en sucres plus solubles permet l'obtention d'un concentré liquide et évite le séchage qui est onéreux. Le pouvoir sucrant de l'hydrolysat est nettement supérieur à celui d'un lactosérum non traité. L'augmentation du pouvoir réducteur des sucres et de leur solubilité constituent des avantages souvent recherchés.

Enfin, les hydrolysats peuvent rentrer dans la composition de nombreux produits alimentaires et permettent d'obtenir différents produits sucrés.

Deux techniques d'hydrolyse semblent particulièrement bien adaptées au cas du lactose contenu dans le lactosérum. La première est l'hydrolyse enzymatique développée par de nombreux auteurs [3, 4, $5,6,7,8,9,10]$. L'enzyme utilisé est la $\beta$-Galactosidase qui est mis soit directement en solution avec le lactosérum, soit fixé sur un 
support poreux, soit incorporé dans un réacteur enzymatique. La seconde est l'hydrolyse acide catalisée par une résine échangeuse de cations.

Ici, après quelques remarques sur la technique d'hydrolyse catalytique appliquée à plusieurs produits différents : lactose, lactosérum liquide ou en poudre déprotéiné et déminéralisé ou non ; nous developpons une étude comparative des deux techniques d'hydrolyse et plus particulièrement des hydrolysats obtenus à partir d'un même lot de lactosérum liquide. D'une part, des observations sont portées sur la présentation des produits d'hydrolyse, leurs qualités organoleptiques, leur comportement aux changements de température et leur aptitude à la conservation, et d'autre part, une méthode d'analyse par chromatographie en phase gazeuse nous permet d'étudier la composition et la teneur des hydrolysats en oligo-saccharides.

\section{MATERIEL ET METHODE}

\section{A. Matières premières}

$1^{\circ}$ Hydrolyse SUR RÉSINES ÉCHANGEuSES D'IONS

- Lactose hydraté $\mathrm{C}_{12} \mathrm{H}_{22} \mathrm{O}_{11}, \mathrm{H}_{2} \mathrm{O}$ (Merck)

- Lactosérum en poudre déprotéiné (Coopérative Laitière de la Prospérité Fermière). Ce produit est obtenu par ultrafiltration du lactosérum liquide puis évaporation sous vide (méthode Spray). Les caractéristiques du produit sont les suivantes :

Humidité : 3 p. 100 ; cendre : 10 p. 100 ; matières azotées : 5 p. 100 ; lactose : 82 p. 100.

- Lactosérum en poudre ou liquide déprotéiné et déminéralisé à 65 p. 100 (Coopérative Laitière de la Prospérité Fermière). Les caractéristiques du produit sont les suivantes :

Humidité : 3 p. 100 ; cendre 2 p. 100 ; matières azotées : 6 p. 100 ; lactose : 88 p. 100 ; acides divers : 1 p. 100.

Le lactosérum liquide est concentré à 18 p. 100 de matière sèche.

2o Hydrolyse PAR VOIE ENZyMatique

— Lactosérum liquide déprotéiné et déminéralisé à 65 p. 100 qui présente les mêmes caractéristiques que le précédent.

- Enzyme Maxilact à 2000 unités d'ortho-nitro-phényl- $\beta$-D galactopyranoside (O.N.P.G.) par gramme.

\section{B. Préparation des échantillons}

Solution $n^{\circ} 1$ : Solution aqueuse à $250 \mathrm{~g} / 1$ de lactose et $60 \mathrm{~g} / 1$ de méso-inositol (témoin interne). 
Solution $n^{\circ} 2$ : Solution de lactosérum liquide déprotéiné et déminéralisé à 65 p. 100 ; concentré à 18 p. 100 de matière sèche.

Solution $n^{\circ} 3$ : Solution à $250 \mathrm{~g} / 1$ de lactosérum déprotéiné en poudre et $60 \mathrm{~g} / 1$ de méso-inositol.

Solution $n^{\circ} 4$ : Solution à $250 \mathrm{~g} / 1$ de lactosérum en poudre déprotéiné et déminéralisé à 65 p. 100 et $60 \mathrm{~g} / 1$ de mésoinositol.

Remarque: La dissolution des produits en poudre se fait à chaud et est suivie d'une agitation à $95^{\circ} \mathrm{C}$ pendant $1 \mathrm{~h}$ qui permet un dégazage du produit.

\section{Principe de l'hydrolyse}

10 Hydrolyse catalytique sur RÉSines Échangeuses d'IONS

La mise au point de la méthode a été effectuée à l'aide de la solution $\mathrm{n}^{\circ} 1$ suivant le schéma ci-dessous.

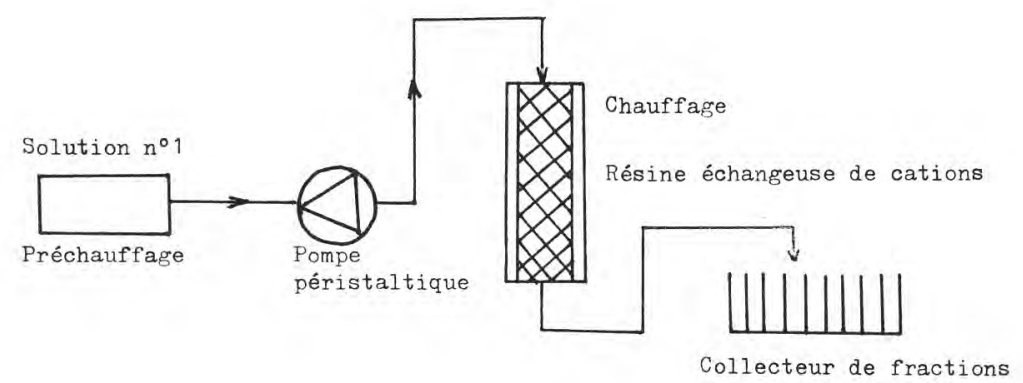

La solution est préchauffée à $95^{\circ} \mathrm{C}$ à l'aide d'un cryostat (Haake FK 2) contenant de l'éthylèneglycol. Ce même appareil thermostatise la colonne d'hydrolyse qui contient la résine échangeuse d'ions du type cationique IR 118 fournie par la Société Applexion*.

Les dimensions de la colonne sont les suivantes :

$\begin{array}{ll}\text { hauteur } & 80 \mathrm{~cm} \\ \text { diamètre intérieur : } & 4,5 \mathrm{~cm}\end{array}$

Le débit de la solution à travers la résine est maintenu constant au moyen d'une pompe péristaltique : $350 \mathrm{~cm}^{3} / \mathrm{h}$.

En sortie de colonne la solution est recueillie par fraction de $9 \mathrm{~cm}^{3}$ à l'aide d'un collecteur de fraction. L'hydrolyse des solutions 2 ,

* Applexion, 4 bis, rue St-Charles - 75015 Paris. 
3 et 4 a été effectuée suivant le même processus. Dans tous les cas, il est nécessaire de déminéraliser totalement le lactosérum à l'aide de résines échangeuses d'ions.

On utilise à cet effet une résine cationique fortement acide du type polystyrène sulfoné XA-60 et une résine anionique moyennement basique du type XA 555 .

\section{Hydrolyse enzymatioue}

L'hydrolyse est effectuée en ajoutant $1,7 \mathrm{~g}$ de Maxilact par kg de matière sèche de la solution $\mathrm{n}^{\circ} 2$. Le mélange est maintenu $24 \mathrm{~h}$ à $20^{\circ} \mathrm{C}$.

\section{Technique d'analyse des sucres par chromatographie en phase gazeuse}

La chromatographie en phase gazeuse (C.P.G.) permet de doser qualitativement et quantitativement en une seule analyse les différents sucres présents dans l'hydrolysat. Elle est donc préférable à la plupart des autres méthodes enzymatiques et colorimétriques qui ne permettent pas un dosage simultané des différents sucres : lactose, glucose, galactose.

\section{1 \% Matériel}

Le chromatographe utilisé est un appareil Packard, modèle 419 équipé d'un détecteur à ionisation de flamme. L'intensité du détecteur est de $2,5 \times 10^{-10} \mathrm{~A}$.

L'injection des échantillons se fait directement en tête de colonne à l'aide d'une seringue S.G.E. de $10 \mu \mathrm{l}$. La colonne spiralée en verre Pyrex a $3 \mathrm{~m}$ de long et $3 \mathrm{~mm}$ de diamètre interne et elle contient de l'OV-17 à 5 p. 100.

Support : Supelcoport $100-120$ mesh.

La température de l'injecteur et du détecteur est de $250^{\circ} \mathrm{C}$.

Débits : gaz vecteur (azote) : $\quad 30 \mathrm{~cm}^{3} / \mathrm{mn}$

hydrogène : $30 \mathrm{~cm}^{3} / \mathrm{mn}$

air $\quad: \quad 300 \mathrm{~cm}^{3} / \mathrm{mn}$

20 Préparation des échantillons : Silylation des sucres

En nous appuyant sur la méthode de Zurcher [11] nous avons mis au point le protocole opératoire suivant :

- prélever $5 \mu \mathrm{l}$ du produit à analyser ;

- évaporer à sec à $80^{\circ} \mathrm{C}$;

- ajouter $50 \mu \mathrm{l}$ de pyridine et $100 \mu \mathrm{l}$ de B.S.T.F.A. à 1 p. 100 de T.M.C.S. ;

- porter à l'étuve $5 \mathrm{~h}$ à $80^{\circ} \mathrm{C}$;

- conserver le dérivé silylé 3 semaines maximum à $4^{\circ} \mathrm{C}$. 
3o Analyse

- température du four : $180^{\circ} \mathrm{C}$ pendant $10 \mathrm{mn}$ puis programmation de $5^{\circ} \mathrm{C} / \mathrm{mn}$ jusqu'à $230^{\circ} \mathrm{C}$;

- injection de 1 à $2 \mu 1$ de l'échantillon ;

- la durée d'une analyse est de $40 \mathrm{mn}$.

\section{RESULTATS ET DISCUSSION}

\section{A. Hydrolyse catalytique sur résines échangeuses d'ions}

Nous avons étudié l'influence de la température, de la concentration en lactose et du débit sur le pourcentage d'hydrolyse d'une solution de lactose pur. En tenant compte de ces données, nous avons mis au point le processus simultané d'hydrolyse de déminéralisation et de décoloration du lactosérum sous ces différentes formes. L'ensemble des résultats obtenus sera publié prochainement.

\section{B. Etude comparative des hydrolysats enzymatiques et catalytiques}

10 Présentation et CONSERVAtion DES hydrolysats

\section{Hydrolysat d'origine enzymatique}

L'hydrolysat de la solution $\mathrm{n}^{\circ} 2$ a été concentré sous vide à 73 p. 100 de matière sèche. Ce pourcentage permet une bonne conservation des produits et évite son altération par les micro-organismes.

L'hydrolysat obtenu est épais et très visqueux, de couleur jaune, son $\mathrm{pH}$ est de 5,3 .

Le produit est sucré et présente des caractéristiques organoleptiques proches du petit-lait.

La coloration évolue avec le temps : après une période de stockage de 4 mois on observe un brunissement très net de l'hydrolysat.

Des essais ont montré que l'hydrolysat enzymatique ne résiste pas à une élévation de température et qu'il s'altère rapidement : réactions de Maillard et caramélisation.

L'agitation nécessaire pour effectuer une dilution avec de l'eau distillée entraîne la formation d'une mousse. On peut attribuer ce phénomène à la présence d'une faible quantité de protéines qui n'a pas été éliminée préalablement.

\section{Hydrolysat d'origine catalytique sur résines échangeuses d'ions}

L'hydrolysat catalytique de la solution $n^{\circ} 2$ est concentré à 75 p. 100 de matière sèche à l'aide d'un évaporateur rotatif sous vide. 
Le produit obtenu est limpide, incolore, et de saveur douce et sucrée. Le pH de la solution est de 7,1 (fig. 1).

Les essais de chauffage n'ont pas entraîné de modification de l'hydrolysat. Ce dernier se conserve dans de bonnes conditions. A température ambiante on note toutefois après $18 \mathrm{j}$, l'apparition d'un léger trouble qui correspond à un début de cristallisation très lente. A $4^{\circ} \mathrm{C}$, ce phénomène est plus rapide et apparaît après $10 \mathrm{j}$ environ.

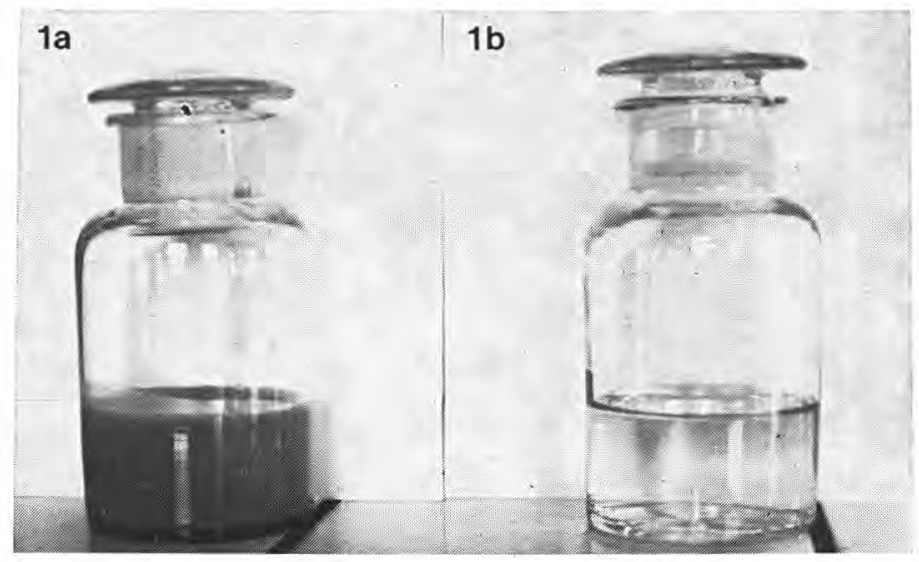

fig. 1

a) Hydrolysat de lactosérum par voie enzymatique.

b) Hydrolysat de lactosérum par voie catalytique sur résines échangeuses d'ions.

L'hydrolyse des solutions 1,3 et 4 conduit à des produits qui présentent les mêmes caractéristiques que l'hydrolysat de la solution 2. Toutefois, les sucres en solution cristallisent quelques heures après la concentration. L'apparition des cristaux de lactose non hydrolysés précède celle des cristaux de glucose et de galactose.

Il faut donc noter ici l'importance de l'échantillon de départ. En effet l'hydrolyse d'un lactosérum en poudre conduit à un hydrolysat dont les sucres cristallisent très rapidement, ce qui n'est pas le cas dans un hydrolysat de lactosérum liquide où la cristallisation est toujours lente, diffuse et incomplète.

La précristallisation du lactose dans la poudre de lactosérum semble donc favoriser sa recristallisation et celle du glucose et du galactose dans l'hydrolysat.

Ce phénomène de cristallisation rapide peut être un avantage si l'on désire récupérer les sucres solides, ou au contraire un inconvénient si l'on veut conserver l'hydrolysat sous forme liquide. 


\section{$2^{\circ}$ COMPOSITION DES PRODUITS}

Cette étude porte sur l'hydrolyse enzymatique et catalytique de la solution $\mathrm{n}^{\circ} 2$.

L'analyse chromatographique de l'hydrolysat enzymatique (fig. 2) permet de déterminer la quantité respective de lactose et de ses

HYDROLYSE DU LACTOSERUM

PAR VOIE ENZYMATIQUE

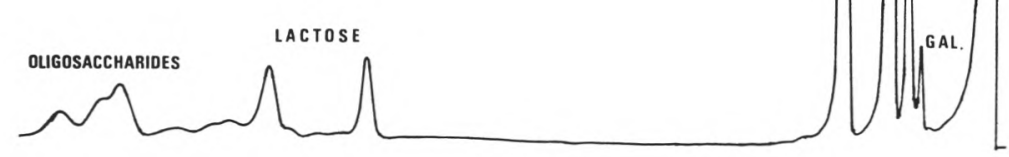

fig. 2

Chromatogramme d'un hydrolysat de lactosérum par voie enzymatique. Condition d'analyse : Colonne OV-17 à 5 p. 100 ; température du four : $180^{\circ} \mathrm{C}$ pendant $10 \mathrm{mn}$; programmation de température de $5^{\circ} \mathrm{C} / \mathrm{mn}$ jusqu'à $230^{\circ} \mathrm{C}$ et un palier isotherme de $20 \mathrm{mn}$. 
produits d'hydrolyse : glucose et galactose. Le pourcentage d'hydrolyse est ici de 75 p. 100.

Sur le chromatographe, on observe également la présence de plusieurs pics qui correspondent à des di et tri-saccharides de structures non identifiées. La concentration de ces produits dans l'hydro-

\author{
HYDROLYSE DU LACTOSERUM \\ PAR VOIE CATALYTIQUE SUR \\ RESINES ECHANGEUSES D'IONS
}

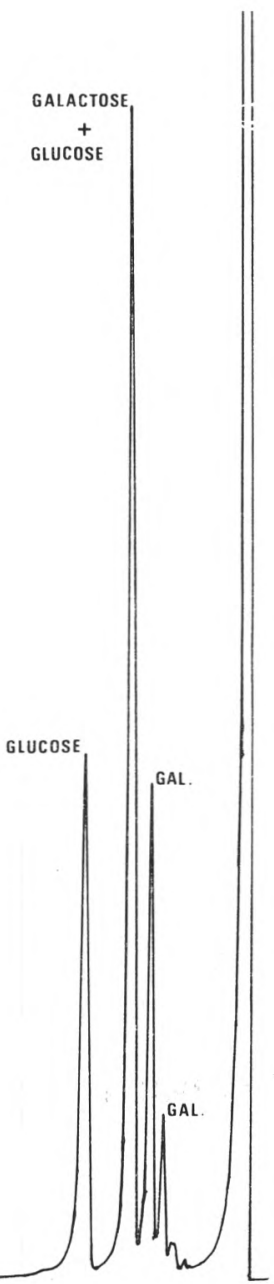

fig. 3

Chromatogramme d'un hydrolysat de lactosérum par voie catalytique, Condition d'analyse : Colonne OV-17 à 5 p. 100 ; température du four : $180^{\circ} \mathrm{C}$ pendant $10 \mathrm{mn}$; programmation de température de $5^{\circ} \mathrm{C} / \mathrm{mn}$ jusqu'à $230^{\circ} \mathrm{C}$ et un palier isotherme de $20 \mathrm{mn}$. 
lysat est non négligeable et comme le signale Roberts et Mc Farren [12], ils ont une influence certaine au niveau du processus métabolique.

L'hydrolysat catalytique est totalement déprotéiné et déminéralisé, son analyse chromatographique (fig. 3) ne met pas en évidence des pics d'oligo-saccharides. Toutefois, une analyse plus fine ne permet pas d'exclure la présence en très faible quantité de ces molécules. Une recherche est en cours à ce sujet.

Cette étude comparative des deux hydrolysats confirme bien les travaux de Wierzbicki [13] qui indiquaient la présence de plusieurs oligo-saccharides dans un hydrolysat enzymatique du lactosérum. Il est donc possible d'affirmer ici que le pourcentage oligo-saccharides est beaucoup plus important dans l'hydrolysat enzymatique que dans l'hydrolysat catalytique.

\section{Ré s u m é}

L'hydrolyse enzymatique et catalytique du lactosérum sur résines échangeuses d'ions conduit à deux produits qui sont sensiblement différents et qui présentent les particularités suivantes.

L'hydrolysat enzymatique est un liquide très épais et visqueux, de couleur jaune avec un pH légèrement acide. Le produit est sucré et présente des caractéristiques organoleptiques proches du petit-lait. Il se conserve plusieurs mois, mais sa coloration s'accentue nettement au cours du vieillissement, et il caramélise facilement à la chaleur.

Quant à l'hydrolysat catalytique, c'est un liquide visqueux, limpide, incolore et inodore, de saveur douce et sucrée avec un $\mathrm{pH}$ voisin de la neutralité. La chaleur n'altère pas le produit. A température ambiante, on peut estimer son temps de conservation de $18 \mathrm{j}$ à l'état limpide, il apparaît ensuite un léger trouble dû à un phénomène de cristallisation extrêmement lent. On remarque que si l'on utilise du lactose ou du lactosérum en poudre, le phénomène de cristallisation est beaucoup plus net et rapide.

L'analyse en chromatographie gazeuse des différents hydrolysats a montré que le pourcentage d'hydrolyse du lactose est sensiblement le même et voisin de 75 p. 100. Par contre on observe que l'hydrolysat enzymatique contient une quantité appréciable de di et tri-saccharides qui n'apparaissent pas dans l'hydrolysat catalytique. Dans ce cas, la présence éventuelle de ces derniers n'est cependant pas à exclure.

Compte tenu des bonnes propriétés de l'hydrolysat catalytique, la mise en place d'une étude sur le développement et l'utilisation industrielle de ce produit semble donc intéressante. 


\section{S u m m a r y}

The enzymatic and catalytic hydrolysis on ion exchange resins of lactoserum yields two rather different products caracterised as follows :

- The enzymatic hydrolysate is a very thick, viscous and yellow liquid with a slightly acid $\mathrm{pH}$. This is a sweet product with organoleptic carateristics very much like whey. It can be kept several months but as it gets older its colour grows darker and it easily caramelizes when heated.

- As to the catalytic hydrolysate, it is a viscous, clear, colourless and odourless liquid, tasting mild and sweet with a $\mathrm{pH}$ near neutral. The product is not altered by heat and at room temperature it can be kept about eighteen days in a limpid state, then it becomes slightly cloudy due to a very slow process of crystallisation. If powdered lactose or lactoserum is used, the crystallisation process is noticeably quicker and more clearly marked.

- The gas liquid chromatography analysis of different hydrolysates has shown that the percentage of lactose hydrolysis is rather the same and near 75 p. 100 . On the other hand it can be noticed that the enzymatic hydrolysate contains a rather high quantity of di and tri-saccharides which do not appear in the catalytic hydrolysate. Yet, in this case, their possible existence is to be considered.

On account of good properties of the catalytic hydrolysate, it should be interesting to set up a study on the development and industrial use of this product.

\section{Remerciements}

L'auteur remercie Mlle Véronique Brouessard pour sa collaboration technique. Il remercie également la Coopérative de la Prospérité Fermière et la Société Applexion pour les produits et les échantillons qu'ils ont bien voulu mettre à sa disposition.

Reçu pour publication en février 1977.

\section{Bibliographie}

[1] (1971). - Lactose intolerance and Milk drinking Habits gastroenterology. $60,4$.

[2] (1972). - Lactose tolerance in a slavic population. Amer. J. of Digestive Diseased, New series, 17, 1.

[3] Borglum (G. B.) and Sternberg (M. Z.) (1972). - Properties of fungal lactase. J. of Food Science, 37, 619-623. 
[4] KosikowsKi (F. V.) and Wierzbicki (L. E.) (1972). - Lactose hydrolysis of raw and pasteurized milks by saccharomyces Lactis lactase. J. of Dairy Science, 56, 1.

[5] Pastore (M.), Morisi (F.) and Viglia (A.) (1972). - Reduction of lactose of milk by entrapped $\beta$-galactosidase. Conditions for an industrial Continuous Process. J. of Dairy Science, 57, 3.

[6] Woyshik (J. H.) and Wondolowski (M. V.) (1973). - Lactose hydrolysis in milk and milk products by bound fungal $\beta$-galactosidase. J. Milk Food Technol, 36, 1.

[7] Rand (A. G.) and LinkLater (P. M.) (1973). - The use of enzymes for the reduction of lactose levels in milk products the Aust. J. of Dairy Technology.

[8] Wierzbicki (L. E.), Edwards (U. H.) and KosikowsKi (F. V.) (1974). - Hydrolysis of lactose in acid whey by lactase bound to porous glass particles in tubular reactors. J. of Food Science, 39.

[9] WeEtall (H. H.) et al. (1974). - The preparation of Immobilized Lactase and its use in the enzymatic hydrolysis of acid whey. Biotechnology and Bioengineering, XVI, 295-313.

[10] Wierzbicki (L. E.) and Edward (W. H.) (1974). - Hydrolysis of lactose in acid whey using $\beta$-galactosidase immobilized on porous glass particles: preparation and characterization of a reasable catalyst for the production of low - lactose dairy product. Biotechnology and Bioengineering, XVI, 397-411.

[11] Zürcher (K.), Hadorn (H.) and Strack (Ch.) (1975). - Gaschromatographische Zuckerbestimmung. Mitt. Gebiete Lebensm. Hyg., 66, 92-116.

[12] Robert (H. R.) and Mc FARREN (E. F.) (1953). - The chromatographic observation of oligoccharadides formed during the hydrolysis of lactose. J. of Dairy Science, 36, 620.

[13] Wierzbicki (L. E.) and Kosikowski (F. V.) (1972). - Formation of oligosaccharides during $\beta$-galactosidase action on lactose. $J$. of Dairy Science, $56,11$. 